\title{
Subfactor theory and its applications - operator algebras and quantum field theory
}

\author{
YASUYUKI KAWAHIGASHI * \\ Department of Mathematical Sciences \\ University of Tokyo, Komaba, Tokyo, 153-8914, Japan \\ e-mail: yasuyuki@ms.u-tokyo.ac.jp
}

\begin{abstract}
We review subfactor theory and its applications to algebraic quantum field theory with emphasis on classification theory.
\end{abstract}

\section{Brief introduction}

My speciality is subfactor theory within theory of operator algebras, and more specifically, I work on studies of mathematical structures appearing in operator algebraic approaches to algebraic quantum field theory. Everyone knows existence of the field of operator algebras, but my experience suggests that rather few know about concrete results and methods in theory of operator algebras, beyond appearance of its name in connection to noncommutative geometry and quantum invariants low dimensional topology. Thus, taking this opportunity, I would like to start with explanations on our aims and methods in operator algebrai theory for non-experts. After that, I will review my recent research results. I refrain from stating precise definitions and exact statements of theorems here, and refer the reader to the references for all such things.

\section{Aims of theory of operator algebras}

Noncommutative geometry of Connes and quantum invariants in 3-dimensional topology, starting with the discovery of the Jones polynomial, are particularly famous among interactions of operator algebra theory and other fields of mathematics. My research is closely related to the latter, so I have written and talked about them on many occasions, but these are rather applications of operator algebra theory and

${ }^{*}$ The author was supported in part by JSPS Grant. 
are not exactly within operator algebra theory in a narrow sense. So before going into such applications, I would like to start with internal aims and techniques within operator algebra theory. I refer the reader to [24] as general textbooks on operator algebra theory.

We study algebras of bounded linear operators on a Hilbert space, which is usually assumed to be separable and infinite dimensional, in theory of operator algebras. We further require that an algebra is closed under the *-operation and an approprite topology. Depending on the choice between the norm topology and the strong operator topology, we obtain a $C^{*}$-algebra and a von Neumann algebra. (The weak operator topology also gives a von Neumann algebra as well as the strong operator topology.) The strong and weak operator topologies are weaker than the norm topology, and closedness under a weaker topology is a stronger condition, needless to say, so a von Neumann algebra is automatically a $C^{*}$-algebra, but methods to study $C^{*}$-algebras and von Neumann algebras are often technically different, so many operator algebraists think that theory of operator algebras consists of two different, but not entirely disjoint, theories of $C^{*}$-algebras and von Neumann algebras. We, of course, have several similarities and interactions between these two, so it is important to know what is happening in the other, and some people work really in the both, but many people work in only one of them, and I work on theory of von Neumann algebras. (Many people outside of theory of operator algebras think that operator algebra theory and $C^{*}$-algebra theory are synonyms, and this is not logically wrong in the above sense that a von Neumann algebra is automatically a $C^{*}$-algebra, but this is against feeling of many operator algebraists.) Here, note that it is very important to assume that an algebra is closed under an appropriate topology. For example, consider a discrete countable group $G$ and its left regular representation $\lambda$. Linear combinations of the unitary operators $\lambda_{g}, g \in G$, make an infinite dimensional algebra, but we need to make a closure in an appropriate topology to obtain a $C^{*}$-algebra or a von Neumann algebra, in order to use a general theory of operator algebras. Some infinite dimensional algebras without completeness, or even a topology, have been often studied in other fields of mathematics, but I feel that a truly interesting structure emerges after a completion. (It is true in many cases that we see interesting structures ready before a completion, and then we do not need to make a completion, but I feel that this is just because we happen to be in a lucky situation.)

The main aim in theory of operator algebras is to understand structures of $C^{*}$ algebras and von Neumann algebras, and thus classification theory is naturally a central topic. That is, we would like to obtain a (computable) complete invariant or a complete enumerative list for operator algebras themselves, their automorphisms, group actions on them, or their subalgebras. In this sense, it would be an ultimate goal to make a complete classification list of all the operator algebras, but such a classification seems to be hopelessly difficult, unfortunately, as well as classification problems for all topological spaces or all Banach spaces. Needless to say, we have many other important problems than the classification problems, such as investigating a property that holds for all or most of operator algebras, determining the class of 
operator algebras for which a certain condition holds, computing (not necessarily complete, but interesting) invariants, and constructing intriguing examples. Free probability of Voiculescu and a series of work on exact $C^{*}$-algebras by Kirchberg are particularly important in connection to such problems, but I limit this review paper to problems directly related to classifications, partly due to my limited taste and knowledge.

An important idea in classification theories of operator algebras is that those in a nice class can be completely classified by an algebraic invariant. Another equally important idea is that we can obtain a simple characterization on which algebraic invariants can indeed arise. These ideas have an origin in a series of stunning work of A. Connes in 1970's and have been sources of many studies. More detailed descriptions of these are given as follows. The conditions for "nice" classes are generally called "amenability". This notion originally appears for a discrete group $G$, and is defined as existence of a nontrivial positive and translation invariant linear functional on $\ell^{\infty}(G)$. (Here positivity of a linear functional means that it maps any positive elements in $\ell^{\infty}(G)$ to a positive number.) Abelian groups and finite groups are amenable. (However, even for the integer group $\mathbf{Z}$, such a linear functional is constructed only with a transcendental method arising from the axiom of choice. It often happens in operator algebra theory that such a transcendental map carries important pieces of information.) For general discrete groups, amenability is a condition "close" to commutativity in a sense. For example, free groups having at least two generators are "the most noncommutative" in the sense that elements never commute except for trivial cases, and they are typical examples of non-amenable groups. A notion of amenability is extended to non-discrete groups easily. We have a notion of amenability for operator algebras as an analogue of this amenability for groups and this condition for operator algebras have many equivalent formulations. The most major achievement of A. Connes within operator algebra theory is a characterization of amenability for von Neumann algebras. That is, he has proved that amenability of a von Neumann algebra is equivalent to hyperfiniteness which means that the algebra can be approximated internally with finite dimensional algebras. (Note that it is often a useful and important idea to pursue an analogue for operator algebras of a notion for groups or fields, based on an idea that groups, rings, and fields are all "similar".) Thus we have important problems such as classification of amenable operator algebras, classification of actions of (not necessarily discrete) amenable groups on amenable operator algebras, and classification of amenable operator subalgebras (after a suitable definition of amenability for inclusions of operator algebras). In the last nearly thirty years, we have seen much great progress on such problems, but still we are far from the completion. The notion of amenability for groups may not be ubiquitous in the entire mathematics, but it is a decisively important condition in operator algebra theory. We have several reasons to believe that unified classification with a simple invariant would immediately fail, if we proceed beyond the amenable class. Also from an operator algebraic viewpoint, an amenable discrete group is "almost like Z", but the other discrete groups are "entirely different". (Dis- 
crete groups of a Lie group, such as $S L(n, \mathbf{Z})$, are important objects in recent theory of operator algebras, but they are very far from being amenable, and few operator algebraic results are known for them. For completely general operator algebras, it is believed that whatever strange condition we may think, anything can happen, as long as it is not obviously prohibited. There is a so-called "Gromov Principle" that any non-trivial statement for general discrete countable groups have a counter-example. Many statements on discrete groups can be translated into those operator algebras, by passing to group algebras, so an analogous statement to this Principle is believed to hold for operator algebras.)

Next I explain what is an "algebraic invariant" in general. By the word "algebraic", I mean that the same or an similar invariant can be defined without topology. We usually consider some maps, such as linear functionals on operator algebras, *homomorphisms from one operator algebra to another, or more complicated maps, and study the set of all such maps with some equivalence relations. It is often the case that the set of equivalence classes is "small" and has some algebraic structure. A typical such example is $K$-theory for $C^{*}$-algebras. "Some equivalence relations" often arise from "trivialization" of inner automorphisms of an algebra, which is of the form $x \mapsto \operatorname{Ad}(u)(x)=u x u^{*}$ for some unitary $u$ in the operator algebra. Examples of "some algebraic structure" are (finitely generated) abelian groups, tensor categories, and ergodic flows. (It may not be appropriate to call the last one "algebraic".) All the classification theorems on von Neumann algebras (and their subalgebras, group actions on them), initiated by Connes and developed by Haagerup, Jones, Ocneanu, Popa, and other people, claim that such algebraic invariants are complete under assumptions of some amenability. In the classification theory of simple separable $C^{*}$-algebras, the relevant algebraic invariants are $K$-theoretic, and many classification theories have been successful along the line of the Elliott conjecture stating that amenable simple separable $C^{*}$-algebras are completely classified by the $K$-theoretic invariants. (We are, however, still far from a complete solution to this conjecture.) Here, the $K$-theoretic invariants are essentially $K_{0^{-}}$and $K_{1}$-groups, but we need some extra related data for the classification.

I also explain basic ideas that are commonly shared in this type of classification theories. A commutative von Neumann algebra is of a form $L^{\infty}(X, \mu)$ and a commutative (unital) $C^{*}$-algebra is of a form $C(X)$, where $(X, \mu)$ in the former is a measure space and $X$ in the latter is a compact Hausdorff space. (If a commutative $C^{*}$-algebra is not unital, we need to consider continuous functions on a locally compact Hausdorff space vanishing at infinity.) Thus, it is an old idea that theories of von Neumann algebras and $C^{*}$-algebras are "noncommutative integration/ergodic theory" and "noncommutative topology", respectively. Noncommutative geometry of Connes is also a recent far-reaching extension of this idea. One important and rather surprising idea in classification theories mentioned above is that passing to noncommutative algebras makes classification statements simpler (although it does not make technical aspects easier at all). For example, consider classification of automorphisms of the hyperfinite $\mathrm{II}_{1}$ factor, which has been one of the most fundamental 
examples of von Neumann algebras since the initiation of the theory by Murray and von Neumann, and is, of course, amenable. If we have two automorphisms $\alpha, \beta$ whose non-trivial powers are never inner, then we have another automorphism $\theta$ and a unitary $u$ in the algebra so that we have $\operatorname{Ad}(u) \cdot \alpha=\theta \cdot \beta \cdot \theta^{-1}$. The inner automorphism $\operatorname{Ad}(u)$ is regarded as a "trivial automorphism", so that a "generic" automorphism, in the sense that no non-trivial powers become trivial, is essentially unique for this operator algebra. This is a great achievement of Connes. A commutative counterpart of an automorphism of the hyperfinite $\mathrm{II}_{1}$ factor in ergodic theory is a measure preserving ergodic transformation of a Lebesgue space with a probability measure, but a statement that all such transformations are conjugate is obviously false. (Actually such transformations are all orbit equivalent, and this fact is important for operator algebra theory, but orbit equivalence loses information which group acts on a Lebesgue space, and this is a problem from a viewpoint of classification of group actions.) It is important that we have $\operatorname{Ad}(u)$ in the above classification theorem of Connes and the fact that a noncommutative algebra can have an inner automorphism not equal to the identity map plays a role in uniqueness here. Furthermore, since commutative $C^{*}$-algebras should be amenable whichever definition we may use, a counterpart of the Elliott conjecture on $K$-theoretic classification of simple amenable $C^{*}$-algebras in the commutative setting would be a statement that (locally) compact Hausdorff spaces are classified up to homeomorphism with $K$-theory, which is, of course, entirely false. Recall that a commutative $C^{*}$-algebras have "many" ideals (unless it is the scalar field $\mathbf{C}$ ), so they are far from being simple. Thus the Elliott conjecture predicts that we have a simple and plain classification statement under an assumption that $C^{*}$-algebras are close to being commutative in terms of amenability, but far from being commutative in terms of simplicity. It has been recently shown that even for amenable simple $C^{*}$-algebras, we have various rather strange phenomena, but still, in comparison to topology, we have a simpler picture after passing to the noncommutative world.

Finally note that a proof of such a classification theorem is always highly technical and relies on improving of approximations step by step while controlling errors.

\section{Subfactor theory of Jones}

Next, I explain how subfactor theory initiated by V. F. R. Jones [13] fits into a general framework as above and why it is related to several "quantum something". I have written and talked on these topics on many occasions, so I will be rather brief and refer the reader to our book [9].

The definition of a simple algebra, of course, requires triviality of two-sided ideals (closed under an appropriate topology). For von Neumann algebras, it has been known that this condition is equivalent to the property that the center is equal to $\mathbf{C}$, and for a historic reason, the terminology "factor" has been used, rather than a "simple von Neumann algebra". Since the days of Murray and von Neumann, studying factors has been a central theme in theory of von Neumann algebras. Then in subfac- 
tor theory, we study the situation where a factor $N$ is included in another factor $M$. Usually, the larger factor $M$ is fixed, so we often write "a subfactor $N \subset M$ ", but we also use the terminology "a subfactor" for meaning the inclusion of $N$ in $M$ in many cases. Again by in idea that groups, rings, and fields are all similar, we consider an analogue of an index of a subgroup and a degree of an extension of a field, and call it the Jones index of a subfactor. This Jones index takes a value in the interval $[1, \infty]$ and can be a non-integer. In this setting, we are interested in an analogue of the Galois theory of extensions of fields and its "quantization". (Usually, one considers a field and its extension in theory of fields, and we can similarly consider here a factor and its extended factor, but in many cases, it is more natural to fix a larger factor and study its subfactors.) A direct analogue of classical Galois theory has been studied in theory of operator algebras for many years as follows. Consider a factor $M$ and an action of a finite group $G$ on $M$ such that any non-trivial element of $G$ acts as a non-inner automorphism of $M$. (Here an idea that an inner automorphism is "trivial" again appears.) We write $M^{G}$ for the fixed point algebra of this action, and then it is automatically a factor and we can recover the group $G$ just from the inclusion $M^{G} \subset G$. More concretely, we consider automorphisms of $M$ that fix all the elements of the subfactor $M^{G}$, and then the original action of $G$ appears. We also have a Galois correspondence between subgroups of $G$ and intermediate algebras of $M$ and $M^{G}$. The Jones index of this subfactor $M^{G} \subset M$ is the order of $G$. It is an important idea in subfactor theory to extend this viewpoint to a general subfactor $N \subset M$ and regard $N$ as a "fixed point algebra" under an action of something like a group. Such an idea was materialized in a very strong form of paragroup theory by Ocneanu [20]. Here, I explain this theory in a form due to Longo and Izumi. Recall that if a subfactor indeed comes from an action of a finite group as a fixed point algebra, then the finite group can be recovered from the subfactor as a certain automorphism group of the larger algebra. For a general subfactor, we consider endomorphisms rather than automorphisms and obtain a certain algebraic system. (By simplicity of $M$, any endomorphism is automatically injective. It is more interesting to consider a non-surjective endomorphism. For such an endomorphism, the image is a proper subalgebra of $M$ that is isomorphic to $M$. Such a case does not occur for finite dimensional algebras, of course, but this easily happens for infinite dimensional algebras.) We have a product operation here, defined as a composition of endomorphisms. We also have an operation of a direct sum as follows. (Note that if we just add two endomorphisms as maps, then the resulting map is not an endomorphism in general.) For two endomorphisms $\rho, \sigma$ of $M$, we define a homomorphism $x \mapsto\left(\begin{array}{cc}\rho(x) & 0 \\ 0 & \sigma(x)\end{array}\right)$ from $M$ to $M \otimes M_{2}(\mathbf{C})$. The image is in $M \otimes M_{2}(\mathbf{C})$ and not in $M$, but if $M$ falls in a class of type III factor that naturally arises in the setting of algebraic quantum field theory that I will explain below, then we have an easy isomorphism from $M \otimes M_{2}(\mathbf{C})$ onto $M$, and using this, we obtain an endomorphism of $M$ that is the direct sum of $\rho, \sigma$. Furthermore, we can define a notion of a dimension for endomorphisms and it takes a value in $[1, \infty]$. This is essentially the same as the square root of the Jones index 
mentioned above. We also have a notion of irreducibility for endomorphisms and if a dimension is finite, we can make an irreducible decomposition of an endomorphism in the unique way in an appropriate sense. The dimension is additive and multiplicative with respect to a direct sum and a composition, respectively, and an algebraic system of endomorphisms behaves similarly to an algebraic system of unitary representations of a compact group where we have a direct sum and a tensor product. For an endomorphism, we have a notion of a conjugate endomorphism which is an analogue of a contragredient representation, and we have a direct analogue of the Frobenius reciprocity for conjugate endomorphisms. We have two differences between our endomorphisms here and unitary representations of compact groups as follows. First, our dimensions are now not necessarily integers. Second, our compositions of endomorphisms are no necessarily commutative, though the tensor product operation, which is the corresponding operation in representation theory, is commutative in an obvious sense. (We have compositions of endomorphisms of an infinite dimensional algebra. There is no reason for such an operation to be commutative.) This algebraic system is a certain tensor category having endomorphisms as objects. We have a notion of amenability for such a category and a tensor category having only finitely many irreducible objects are amenable, as an analogue of a finite group. In such a case, we say that a category is rational. The rules of irreducible decompositions of compositions of two irreducible objects are called the fusion rules. By comparing two ways of irreducible decompositions of a product of three objects, we obtain $6 j$-symbols, as in representation theory of compact groups. We simply call them $6 j$-symbolsi, or quantum $6 j$-symbols. A paragroup is an abstract algebraic system and axiomatized with several properties of such categories of endomorphisms like those on fusion rules and quantum $6 j$-symbols. Popa's classification theorem says that if we have amenability for both factors and paragroups, then subfactors are completely classified by paragroups. We omit details on these categories, quantum $6 j$-symbols, and paragroups and refer the reader to the book [9] for precise definitions. Here we only make brief remarks, in comparison to similar structures appearing in conformal field theory and quantum groups, that the fusion rules here are not necessarily commutative and that the (quantum) dimensions are always positive now. Here we also remark that if a tensor category arising from a subfactor as above has only finitely many irreducible objects, then we can construct a complex-number valued topological invariant of 3dimensional oriented closed manifolds, and more generally, 3-dimensional topological quantum field theory from such a category. This is a construction of Ocneanu by generalizing the one by Turaev-Viro. (Note that a category here does not have a braiding in general. We also have operator algebraic methods to produce a braiding.)

Now Popa's classification theorem mentioned above falls in a general principle, explained in the above section, that an algebraic invariant becomes complete under amenability, and in this sense, the result itself is similar to other classification theorems, but we now have a new feature as follows. A very nice abstract characterization of paragroups has been obtained by Ocneanu, but it is not clear at all what examples of paragroups we indeed have, and this problem can be studied within in 
a framework of operator algebras. On one hand, finitely generated abelian groups and ergodic flows, which appear as invariants of operator algebraic objects, have been studied well for a long time. On the other hand, a history of studies of tensor categories is relatively short, and not much has been known beyond representation theories of groups and quantum groups having deformation parameters. We, however, think, from a viewpoint of theory of subfactors, that tensor categories arising from quantum groups with a deformation parameter $q$ are special, we have a great deal of other tensor categories, and operator algebras are useful for studies of them. (The biggest evidence for such an idea is a combinatorial study of Haagerup $[12,1]$ for subfactors with small Jones index. He made an exhaustive search for paragroups in a very limited index range, found a large list of candidates that do not seem to be related to usual quantum group theory, and proved that two of them are indeed realized after very hard computations.) So, even if one is interested only in tensor categories and topological invariants arising from them, not in operator algebras themselves, one can still hope for obtaining new results not known in other fields with operator algebraic mathods. One informal reason for such possibility is that infinite dimensional operator algebras are so "large" that one can put many mathematical objects into them. In the case of operator algebraic invariants such as finitely generated abelian groups and ergodic flows, we do not obtain really new examples through operator algebras, so the new situation for tensor categories is different from these predecessors and interesting for this reason. This is a basis for a series of new relations between subfactor theory and "quantum something".

\section{Algebraic Quantum Field Theory}

There have been different mathematical approaches to quantum field theory, and here I explain the one based on operator algebras. This approach relies on algebras of bounded linear operators, rather than operator valued distributions, and is called "algebraic" quantum field theory in this sense. A standard textbook is [11], and one can look at a proceedings volume [18] for recent developments. Here I present a basic setting below.

We fix a "spacetime" and consider appropriate bounded regions in it, those called double cones for example. To each such a region $\mathcal{O}$, we assign a von Neumann algebra $A(\mathcal{O})$ generated by physical observables in $\mathcal{O}$. (We are now in the realm of quantum mechanics, so physical quantities are represented as operators on a Hilbert space.) We have a fixed Hilbert space for all $\mathcal{O}$ 's. Mathematically speaking, this gives a family of operator algebras parameterized by $\mathcal{O}$ 's. We require a set of physically natural axioms to hold for such a family of operator algebras and study such families mathematically. Here I explain only the fundamental axioms, though we often assume various sets of extra axioms in concrete studies. First, we assume that we have a larger operator algebra for a larger spacetime region $\mathcal{O}$, which means that we have more observable quantities in a larger region. Second, if two regions $\mathcal{O}_{1}, \mathcal{O}_{2}$ are spacelike separated, then two operator algebras $A\left(\mathcal{O}_{1}\right), A\left(\mathcal{O}_{2}\right)$ commute, which is 
called locality. This means a relativistic requirement that if one cannot reach $\mathcal{O}_{1}$ from $\mathcal{O}_{2}$ even with a speed of light, then we have no interactions between them, and thus physical quantities in these two regions commute. Next we assume that we have a projective unitary representation $u_{g}$ of a group of "spacetime symmetry" on the Hilbert space and $u_{g} A(\mathcal{O}) u_{g}^{*}=A(g \mathcal{O})$ holds, which is called covariance. A physically natural choice of the spacetime symmetry is the inhomogeneous Lorentz group of the 4-dimensional Minkowski space, but we will also consider other choices of the symmetry below. We also have axioms on a vacuum vector and positivity of energy, but I omit explanations on them here. We often call a family $\{A(\mathcal{O})\}$ of operator algebras a net of operator algebras, because the index set of double cones $\mathcal{O}$ is directed with respect to inclusions.

Now we have a mathematical problem of studying structures of such nets of operator algebras under a suitable set of axioms and even classifying them with a certain algebraic invariant. Our algebraic invariant is representation theory of a net of operator algebras. Suppose we have a net $\{A(\mathcal{O})\}$ of operator algebras. These operator algebras act on a Hilbert space from the beginning by definition, but we also consider their representations on other Hilbert spaces. The original Hilbert space has a special vector called a vacuum vector by an axiom, so the original representation on this Hilbert space is called a vacuum representation. We then consider a compatible family of representations of the algebras $\{A(\mathcal{O})\}$ with covariance property on another Hilbert space, without requiring a vacuum vector there, and study unitary equivalence classes of such representations. We further have notions of a direct sum, irreducible decomposition, a tensor product, and a dimension for such representations. Note that we now have representations of a family of algebras, so the notion of tensor product is not obvious at all here. (In quantum group theory, they have a coproduct, but we do not have such an operation here.) Also, considering a dimension in the ordinary sense is meaningless since it is always infinite. These difficulties are overcome with the Doplicher-Haag-Roberts (DHR) theory [8]. That is, one first shows that any representation, up to unitary equivalence, is realized as an endomorphism of a net of operator algebras, based on the Haag duality, which is a stronger form of locality. (Note that an endomorphism of a net of operator algebras, appropriately defined, gives a new representation on the original Hilbert space. The DHR theory says that any representation can be put in this form after a (possible) change within the unitary equivalence class.) Then the operation called a "tensor product" is defined as a composition of two endomorphism and a dimension is also defined nicely. It is obvious that this situation is similar to that in subfactor theory explained in the previous section. Actually, the DHR theory is much older than subfactor theory of Jones, and the relations between the two were clarified by Longo [17]. In the previous section, we saw that the composition operation of endomorphisms is not commutative, but here, due to locality in the axioms, we have commutativity of composition, up to unitary equivalence. In this way, we obtain a tensor category of representations of a net of operator algebras.

As I mentioned above, the most natural choice for the spacetime would be the 
4-dimensional Minkowski space. Many deep results have been obtained for this setting, but in connection to "quantum something", we have interesting mathematical structures on spacetime of lower dimensions, such as 1, as in [10], for example. One dimensional case is not a "spacetime" any more, but any dimension is allowed in axiomatic mathematical studies, and for the case of one space dimension and one time dimension, which is quite interesting both mathematically and physically in the context of conformal field theory, a net of operator algebras on the 2-dimensional Minkowski space is decomposed into a tensor product of two nets of operator algebras on one-dimensional spaces in an appropriate sense, and such nets are called chiral nets and well-studied. One might suspect that problems in lower dimensions, particularly in dimension one, are mathematically easier, but this is not the case. For nets of operator algebras on a one-dimensional space, one take non-empty bounded open intervals as $\mathcal{O}$ in the above, but then a subtle and interesting problem, which does not occur in dimension three or higher, arises from the fact that its complement is not connected. For example, a composition of two endomorphisms arising from representations is commutative up to unitary equivalence, as mentioned above, and this unitary operator producing the unitary equivalence in the one-dimensional setting brings a braiding. In this way, the representation category becomes braided. In dimension 3 or higher, this braiding automatically becomes trivial, but many nontrivial examples of interesting braiding happen in the one-dimensional setting and braided tensor categories corresponding to the Wess-Zumino-Witten models are realized. Also, the dimensions of representations are automatically integers in a higher dimensional spacetime, but we have non-integer dimensions of representations for nets of operator algebras on a one-dimensional space and they are counterparts of quantum dimensions in quantum group theory.

\section{Tensor category, modular invariant, $\alpha$-induction}

I have finally come to my own research topics. The contents of this section describe what I have been studying after writing the book [9]. I refer the reader to references for all technical matters, and briefly mention the main results.

\subsection{Completely rational AQFT}

As explained above, a net of operator algebras on one-dimensional "spacetime" produces a braided tensor category, but in connection to conformal field theory and topological quantum field theory, we are interested in tensor categories which have only finitely many irreducible objects and a non-degenerate braiding. The finiteness condition is usually called rationality. The non-degeneracy of a braiding is equivalent to invertibility of the $S$-matrix, and a braided tensor category with this non-degeneracy and rationality is called a modular tensor category. See [25] for details on this notion. Our main results in [16] are that we have a simple operator algebraic sufficient condition for the rationality condition of the representation category and that the braiding 
is automatically non-degenerate under this condition. We call this operator algebraic condition complete rationality. By results in [26], [28], we know that the nets of operator algebras arising from loop groups of $S U(n)$ [22] are completely rational. Xu further studied completely rational nets of operator algebras in detail in [29, 30, 31]. Roughly speaking, complete rationality is preserved under various operations for nets of operator algebras.

\section{$5.2 \alpha$-induction and modular invariant}

As mentioned above, representation theory of nets of operator algebras has much similarity to that of compact groups. A method of $\alpha$-induction gives a machinery in representation theory of nets of operator algebras, analogous to induction and restriction in the ordinary representation theory. As an analogy of a pair of a group and its subgroup, we consider a pair of a net of operator algebras and a net of subalgebras, and we produce a representation of a larger net from that of a smaller net. We use the name "induction" here because we pass from smaller algebras to larger algebras, but in the setting of nets of operator algebras, a larger net has a "smaller symmetry", so this is more like a restriction operation in some sense. This construction of $\alpha$-induction was first defined in Longo-Rehren [19], and many interesting properties and examples were found by $\mathrm{Xu}$ [27]. Böckenhauer-Evans [2] further studied this construction. Around the same time as Longo and Rehren, Ocneanu [21] has made a combinatorial study on the $A-D-E$ Dynkin diagrams from a quite different motivation. In [3], we have proved that both definitions of LongoRehren and Ocneanu can be generalized to a situation where we have a braiding in the sense of Rehren [23] and we have a unified definition there. By combining both ideas, we have obtained several results in $[4,5,14]$. We would like to construct a representation of a larger net of operator algebras from athat of a smaller net, and use a braiding in the $\alpha$-induction method. A braiding always comes in a pair of an overcrossing and an undercrossing, and depending on these, we have two kinds of $\alpha$ induction. We distinguish them by putting the \pm symbol. Actually, we cannot make a genuine representation of a larger net from that of a smaller net in general, and we obtain only a "quasi"-representation in some sense. If we take the intersections of the "quasi"-representations arising from $\alpha$-induction with a positive braiding and those with a negative braiding, then it exactly gives the representations of the larger net. If the representation category fo the smaller net is modular (as in the completely rational case, for example), then we have a finite dimensional unitary representation of $S L(2, \mathbf{Z})$, and we can produce a matrix with non-negative integer entries in the commutant of the range of this unitary representation by using positive and negative $\alpha$-inductions. This was proved in [3] under a very general assumption based on works of Ocneanu and Böckenhauer-Evans [2]. Such matrices have been studied well in general under the name of "modular invariant" and several classification theorems, starting with [6], have been obtained. (See [7] for example.) Our results enable one to interpret and realize such classification results with operator algebraic methods. Much has been studied about tensor categories arising from $\alpha$-induction which do 
not have a braiding in general.

\subsection{A central charge and classification of nets of factors}

I recall a basic idea for classification is that we should have simple complete algebraic invariants for the amenable case. For nets of operator algebras, each von Neumann algebra $A(\mathcal{O})$ assigned to a spacetime region $\mathcal{O}$ is always isomorphic to the unique hyperfinite type $\mathrm{III}_{1}$ factor, an amenable factor, under the standard set of axioms, so the isomorphism class of each algebra is useless for classification of nets. We expect that representation theory for completely rational nets on the one-dimensional space would give a complete invariant, but no results of this type have been obtained. It is also totally unclear that which modular tensor category arises as a representation category of a net of operator algebra. All the constructions so far have been obtained depending on the case. Thus the general classification and construction problems are very far from a satisfactory solution, but we have recently obtained a first classification theorem in this direction in [15] as follows. We consider nets of operator algebras on the one-dimensional space, and we compactify the space to $S^{1}$. (This is just for simplicity of various descriptions. We could work on $\mathbf{R}$ without compactification if we want. We also take the orientation preserving diffeomorphism group of $S^{1}$ as the group of "spacetime" symmetries. (It is not mathematically clear how strong this assumption is, but we have many interesting examples satisfying this condition.) Then we can define a real number, called the "central charge", for a net of operator algebras through representation theory of the Virasoro algebra. It is well-known that if this central charge $c$ is less than one, then it can take only values in a discrete series, and our main results in [15] say that in such a case, we have a complete classification and listing of nets of operator algebras. We fully use theories of $\alpha$-induction and modular invariants mentioned above. The classification result is described with pairs of the $A-D-E$ Dynkin diagrams.

\section{$6 \quad$ Future developments}

Classification of nets of operator algebras still has many open problems, such as finding a complete invariant, characterizing possible invariants, and listing all the possible nets. We also have various problems on relations among extra axioms in addition to the standard set of axioms. Furthermore, we have limited (or no) knowledge on its relations to quantum groups, vertex operator algebras, the monster group, the moonshine, and topological quantum field theory. I finish this survey with a hope of further developments on these and yet-unknown directions.

\section{References}

[1] M. Asaeda \& U. Haagerup, Exotic subfactors of finite depth with Jones indices $(5+\sqrt{13}) / 2$ and $(5+\sqrt{17}) / 2$, Comm. Math. Phys. 202 (1999) 1-63. 
[2] J. Böckenhauer \& D. E. Evans, Modular invariants, graphs and $\alpha$-induction for nets of subfactors I, Comm. Math. Phys. 197 (1998) 361-386. II 200 (1999) 57-103. III 205 (1999) 183-228.

[3] J. Böckenhauer, D. E. Evans \& Y. Kawahigashi, On $\alpha$-induction, chiral projectors and modular invariants for subfactors, Comm. Math. Phys. 208 (1999) 429-487.

[4] J. Böckenhauer, D. E. Evans \& Y. Kawahigashi, Chiral structure of modular invariants for subfactors, Comm. Math. Phys. 210 (2000) 733-784.

[5] J. Böckenhauer, D. E. Evans \& Y. Kawahigashi, Longo-Rehren subfactors arising from $\alpha$-induction, Publ. Res. Inst. Math. Sci. 37 (2001) 1-35.

[6] A. Cappelli, C. Itzykson \& J.-B. Zuber, The A-D-E classification of minimal and $A_{1}^{(1)}$ conformal invariant theories, Comm. Math. Phys. 113 (1987) 1-26.

[7] P. Di Francesco, P. Mathieu \& D. Sénéchal, "Conformal Field Theory", SpringerVerlag, Berlin-Heidelberg-New York, 1996.

[8] S. Doplicher, R. Haag \& J. E. Roberts, Local observables and particle statistics, I. Comm. Math. Phys. 23, 199-230 (1971); II. 35, 49-85 (1974).

[9] D. E. Evans \& Y. Kawahigashi, "Quantum Symmetries on Operator Algebras", Oxford University Press, Oxford, 1998.

[10] K. Fredenhagen, K.-H. Rehren \& B. Schroer, Superselection sectors with braid group statistics and exchange algebras, I. Comm. Math. Phys. 125 (1989) 201226; II. Rev. Math. Phys. Special issue (1992) 113-157.

[11] R. Haag "Local Quantum Physics", Springer-Verlag, Berlin-Heidelberg-New York, (1996).

[12] U. Haagerup, Principal graphs of subfactors in the index range $4<3+\sqrt{2}$, in "Subfactors - Proceedings of the Taniguchi Symposium, Katata -", (ed. H. Araki, et al.), World Scientific, (1994), 1-38.

[13] V. F. R. Jones, Index for subfactors, Invent. Math. 72 (1983) 1-25.

[14] Y. Kawahigashi, Generalized Longo-Rehren subfactors and $\alpha$-induction, Comm. Math. Phys. 226 (2002) 269-287.

[15] Y. Kawahigashi \& R. Longo, Classification of Local Conformal Nets. Case c $<1$, to appear in Ann. Math., math-ph/0201015.

[16] Y. Kawahigashi, R. Longo \& M. Müger, Multi-interval subfactors and modularity of representations in conformal field theory, Comm. Math. Phys. 219 (2001) 631-669. 
[17] R. Longo, Index of subfactors and statistics of quantum fields, I. Comm. Math. Phys. 126 (1989); II. 217-247 \& 130 (1990) 285-309.

[18] R. Longo (ed.), "Mathematical Physics in Mathematics and Physics", Fields Inst. Commun. 30, Providence, Rhode Island: AMS Publications.

[19] R. Longo \& K.-H. Rehren, Nets of subfactors, Rev. Math. Phys. 7 (1995) 567597.

[20] A. Ocneanu, Quantized group, string algebras and Galois theory for algebras, in Operator algebras and applications, Vol. 2 (Warwick, 1987), (ed. D. E. Evans and M. Takesaki), London Mathematical Society Lecture Note Series 36, Cambridge University Press, Cambridge, 1988, 119-172.

[21] A. Ocneanu, Paths on Coxeter diagrams: from Platonic solids and singularities to minimal models and subfactors, (Notes recorded by S. Goto), in Lectures on operator theory, (ed. B. V. Rajarama Bhat et al.), The Fields Institute Monographs, AMS Publications, 2000, 243-323.

[22] A. Pressley \& G. Segal, “Loop Groups”, Oxford University Press, Oxford, 1986.

[23] K.-H. Rehren, Braid group statistics and their superselection rules, in: "The Algebraic Theory of Superselection Sectors", D. Kastler ed., World Scientific, Singapore, 1990.

[24] M. Takesaki , "Theory of Operator Algebras. I, II, III" Springer-Verlag, BerlinHeidelberg-New York, 2002.

[25] V. G. Turaev, "Quantum Invariants of Knots and 3-Manifolds", Walter de Gruyter, Berlin-New York, 1994.

[26] A. Wassermann, Operator algebras and conformal field theory III: Fusion of positive energy representations of $S U(N)$ using bounded operators, Invent. Math. 133 (1998) 467-538.

[27] F. Xu, New braided endomorphisms from conformal inclusions, Comm. Math. Phys. 192 (1998) 347-403.

[28] F. Xu, Jones-Wassermann subfactors for disconnected intervals, Comm. Contemp. Math. 2 (2000) 307-347.

[29] F. Xu, Algebraic coset conformal field theories I, Comm. Math. Phys. 211 (2000) $1-44$.

[30] F. Xu, 3-manifold invariants from cosets, preprint 1999, math.GT/9907077.

[31] F. Xu, Algebraic orbifold conformal field theories, Proc. Nat. Acad. Sci. U.S.A. 97 (2000) 14069-14073. 